\title{
Computer Numerical Control Machine Tool Scram Controls Principle Analysis and Fault Diagnosis
}

\author{
Lijuan Shi ${ }^{1, a}$, Jinying Chen ${ }^{2, b}$ ZhaoKun $\mathrm{Li}^{3, \mathrm{c}}$ Huamei Bian ${ }^{4, \mathrm{~d}}$ \\ ${ }^{1}$ Beijing Industry Vocational \& Technical college, Beijing 100042, China \\ ${ }^{2}$ Beijing Industry Vocational \& Technical college, Beijing 100042, China \\ ${ }^{3}$ Beijing Industry Vocational \& Technical college, Beijing 100042, China \\ ${ }^{4}$ Beijing Industry Vocational \& Technical college, Beijing 100042, China \\ asljzch_2000@126.com, ${ }^{\mathrm{b}}$ vegachjy@163.com, \\ c2008lizhaokun@163.com, ${ }^{\mathrm{c}}$ bianhuamei0@163.com
}

\begin{abstract}
Keywords: FANUC OI-MC NC System, Emergency stop control circuit, Emergency stop fault, Emergency stop fault diagnosis process

Abstract. In the control of CNC machine tools, the emergency stop control is essential, and it plays an important role in the operation of the whole $\mathrm{CNC}$ machine tool. By analyzing the scram control principle of FANUC OI-MC CNC system, this thesis is designed to put forward the diagnosis method of emergency stop hardware signal fault and to provide reference for fault diagnosis of CNC machine tools by taking the actual fault analysis and treating process into consideration.
\end{abstract}

\section{Introduction}

Usually, there is a scram button on the machine operator panel and the handheld unit of the CNC machine tools, which plays an important role in the control of CNC machine tools. For instance, when program coordinate calculation error occurs and knife fault is about to happen, pressing the scram button can avoid causing damages to mechanical components; pressing the scram button before CNC system shuts down can avoid glides by self-gravity of the Z-axis. That is to say, scram button is designed for protecting people and machine from danger in emergencies. After pressing the scram, the system will be halted and all the feed shafts and principal axis will enter the status of on-positon; some of the servo drive high voltage power supply circuits will be cut down. Only when the trouble has been removed, people unscrew the emergency stop button, and the numerical control system will return to normal. In practical use, it is common to find the trouble unresolvable, but only when it is solved can restart the machine into normal use. Scram control of FANUC 01 CNC system is the first machine tool protection signal that needs to be dealt with to ensure the smooth function of the interruption system in emergency during the adjustment process. There are two types of emergency stop signal * ESP (emergency stop): software signal, which is G8.4, and hardware signal, which is X8.4. CNC machine tools directly read signal X8.4 and PMC input signal G8.4. When either one of the two signals the signal is low, the system will alarm. This paper constructs fault analysis towards the hardware-related emergency stop alarm signal X8.4 control principle and its failure.

\section{Scram Controls Principle}

$\mathrm{X} 8.4$ is hardware signal of CNC system emergency stop alarm. It links to a control panel installed on the handheld unit or an emergency stop button, and forms an emergency stop signal X8.4 control loop by connecting with the feed axis limit switches,. Its electrical emergency stop control circuit is connected as shown in Fig. 1. 

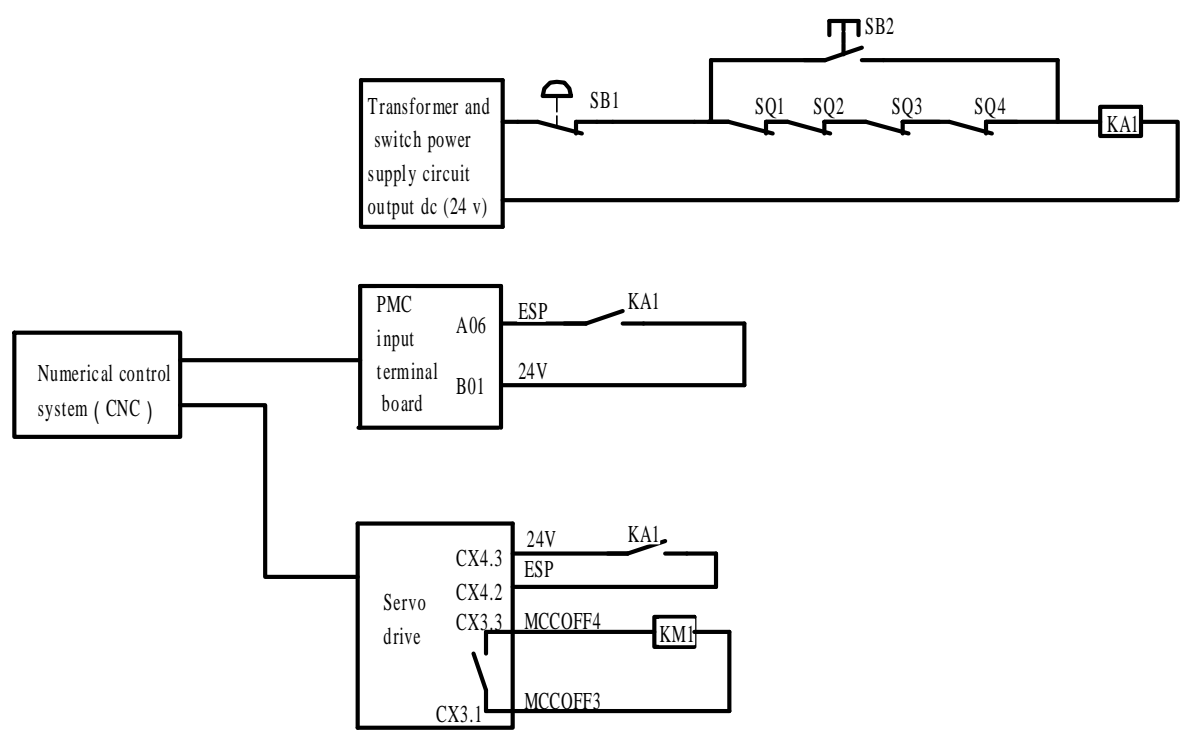

Fig.1 FANUC ESP connection of NC System

In fig.1, SQ1-SQ4 respectively indicates the positive and negative direction of the $\mathrm{X}$ and $\mathrm{Z}$ axes. As can be seen in the figure, the ESP button and the four limit switches are in series to control the KA relay coil which controls the ESP of the machine. Under normal circumstances, the ESP button is in unscrew status, the feed axis is not in the overrun state and KA1 coil is in the power on state. The normally open contact of the KA1 relay which is associated with the PMC input terminal board and servo drive device is closed. The CNC operates normally. And it output the enable signal of the servo drive unit through the PMC and the KM1 contactor coil powers on. The driving device powered on realized by the three main contacts and the machine tool enters into the working state. Conversely, when the ESP button is pressed, if each feed axis of the machine tool is in overrun position, then the KA1 relay powers off. If the normally open contact of the KA1 relay which is associated with the PMC and servo drive device is open, CNC system thinks that there is an external failure, cannot output drive devices enable signal, the servo drive device cannot power on, while it shows "ESP" alarm on the monitor and the whole machine tool is in the emergency stop state. The SB2 button in the picture above is used to remove the over travel state when the system is in the state of overrun.

\section{Scram Fault Diagnosis Methods}

Through the research of ESP control circuit principle, we can know that the emergency stop fault analysis and diagnosis can be divided into the following aspects: View ladder diagram, view point definition and state, judge the ESP button, limit position switches, relays and other electrical components' state. When faced with the ESP alarm about X8.4, the process of fault diagnosis is as shown in figure 2. 




Fig.2 X8.4 ESP signal fault diagnosis flow chart

As can be seen in the figure 2, when the ESP alarms, we first apply the PMC ladder diagram and the point position of the NC system to see whether the X8.4 signal is high. If it is high level, then it means G8.4 signal lead the warning. If the X8.4 is low, then check whether the KA1 relay coil is powered on. We can use a universal meter to detect whether the coil voltage is normal, to determine whether the relay is normal. If it is not a relay fault, we can use the meter of the beeps to detect and determine the fault location of the emergency stop circuit.

\section{Emergency stop fault case analysis}

As can be seen from Figure 2, the failures related to the X8.4 emergency stop signal are normally as following:

1)The terminal's voltage of ESP hardware signal X8.4 is 0V and the signal of X8.4 is low

As can be seen in figure 1 and fig. 2 , the reason of the terminal's voltage of ESP hardware signal $\mathrm{X} 8.4$ is $0 \mathrm{~V}$ maybe the hardware failures on the ESP button, limit switches, relays and I/O board. We need to check and judge whether the hardware contactor is normal and whether the components are damaged.

2) The terminal's voltage of ESP hardware signal X8.4 is $24 \mathrm{~V}$ and the signal of X8.4 is low

As can be seen from Figures 1 and 2, when the hardware emergency stop signal X8.4 terminal voltage is $24 \mathrm{~V}$, it indicates peripheral machine circuit consisting of the emergency stop button and limit switch emergency stop has no problem, it may be I / O boards problem.

\section{Emergency stop invalid failure analysis}

Symptom: a phenomenon occurs when CNC lathe FANUC OI-MC system turns on: the machine is in the "stop" state, unscrew the "stop" button on the control surface and the hand-held unit, "stop" warning can not be released, emergency stop buttons are invalid.

Analysis process: 1) the "Push" and " Turn on" the emergency stop button on the control panel and the hand-held unit, while viewing the PMC point position state diagram or ladder diagram, check if the hardware signal X8.4 conversion between "low" level and "high" level occurs. If so, then it indicates that there is no problem with X8.4 loop it may be the problem of G8.4 signal.Check and make sure that X8.4 of signal isn't changed; 2) the "Push" and " Turn on" the emergency stop button on the control 
panel and the hand-held unit to check if the KA1 relay coil is energized. By checking if the relay LEDs light, at the same time $24 \mathrm{~V}$ voltage wasn't been detected by multimeter coil, which can determine it is the fault of emergency stop circuit; 3)By checking the warning alarm, no overtravel alarm which suggests that the limit switch has no problem, normally by checking the emergency stop button, it is found that the normally- closed contact point of emergency stop button on a handheld unit is disconnected when pressing down, and still in a disconnected state when it is closed, eliminate faults in a proper order, and found the fault is because of the circuit disconnection . Reconnect the circuit, and fault is released.

\section{Fault analysis of overrun alarm cannot be released}

Fault phenomenon occurs after turning on the NC system, lathe is in Emergency Stop state while "Z axis positive overtravel" alarms,. After pressing the button of overtravel releasing, the $\mathrm{Z}$ axis moves to the negative direction, but the alarm cannot be released.The phenomenon of these faults may be related to the limit switches on the feed axis and the "super lift" button on the operating panel. The process can be analyzed as follows: 1 ) Press the "super lift" button while viewing PMC ladder or point state diagram to find whether the position signal which is related to super lift button SB2 is changed. If there is no change, it may be the button' s fault or button circuit' s fault. if the change occurs, it may be the limit switch problem, or the ladder diagram' s problem. After inspection, we find that the position signal related to the button is normal.2 ) Press the "super lift" button, the $\mathrm{Z}$ axis will move out of the super range in the negative direction, but the machine still displays the "super range alarm", thus we can determine that the limit switches may have problems. We can check the limit switches on the machine and find that the normally closed contact was broken after the collision with the hard limit block. Thus it can' $t$ reset. After changing the limit switch, the fault is eliminated.

\section{Power line and control line coil of servo drive power off}

Fault phenomenon: After the start of the NC system, ESP alarms, the supply voltage of servo drive device is zero and MCC control loop of the contactor did not suck.

From Figure 1 and the servo drive connections of the Fanuc 0I-MC system we can know, when the servo drive device of the machine is in the "reset state" and the driving device have no problems, the power-line-control-coil KM1 powers on and the contact closed, meanwhile the feed drive device is powered on. If one of the conditions is not satisfied, the driving device cannot power on and the machine is in a "ESP" state. Therefore, the failure can be carried out as follows :

1 ) After the CNC system is started, we can detect the KM1 coil voltage by a universal meter, if it is $24 \mathrm{~V}$, it means that the contactor is broken. if it is not $24 \mathrm{~V}$, we need to see whether the drive device has alarm and ESP control circuit. After testing the KM1 coil does not have.

2 ) We can find that the drive device have no alarm signals and the relay' s coil has powered on, then we can judge that the CX4.3 has problem.

3 ) The relay contact which closed to the interface has fault. The contact voltage is normal after measuring, but the normally open contact between $24 \mathrm{~V}$ pin (CX4.3) and KA1 is disconnected, leading that the machine is in ESP state and the servo drive device for power line control coil powers off.

\section{Conclusion}

Regarding to the scram stop fault diagnosis and analysis, first of all, it needs to be familiar with and master different ESP control principle of the different NC system. It can be understood its control process by viewing the schematics and PMC ladder diagram of the machine tool electric, then analyze the reason of the fault diagnosis and elimination. ESP failure generally is caused by the emergency stop 
button not normal reset, super range, servo alarm and the principal axis alarm. In the application of appropriate method to exclude the ESP button and limit switch fault and X8.4 fault, it can be analysis the reasons of emergency stop according to the PMC ladder. Using PMC state point monitoring and dynamic ladder diagram to check which conditions are not met, resulting in G8.4 is not connected. Then using the detection instrument or other measures to detecting the reasons of the failure on the machine tools and rapid troubleshooting. In conclusion, for the diagnosis and analysis of scram stop failure is analyzing the reason cause the hardware signal X8.4 and the software signal G8.4 to low level.

\section{Acknowledgment}

This research was supported by Beijing areal Project of Ministry of Education(KM2016-1085-3003), Collegestudents' scientific research innovation(bgzykey201615)and Beijing Polytechnic College in 2016(bgzykey201615,bgzykyt201617).

\section{References}

[1]Qiujun WU , Jianjun WANG . FANUC 0I Emergency Stop Fault Diagnosis and Analysis of NC machine tool [J]. Coal Mine Machinery , 2014,35 ( 04 ) : 247-248.

[2]Xingguo ZHONG. Fault Diagnosis and Maintenance of Emergency Stop and Alarm in NC System [J]. Machine Tool\&Hydraulics, 2012,42(18):129-131.

[3]Zhi ZHENG. The Fault Diagnosis and Maintenance of CNC Machine Tools [J]. Coal Mine Machinery , 2013,34 ( 03 ) : 295-296.

[4]FANUC CNC Maintenance and Adjustment (OI-MD) Tutorial [M].Beijing : Higher Education Press.

[5]Lan ZHOU , Shaoai CHEN. Connecting and Debugging of NC System and PMC Programming [M].Beijing : China Machine PRESS , 2012.

[6]Caiying HE , Jinpeng YANG. Fault Diagnosis and Maintenance of CNC Machine Tool with Emergency Stop and Super Range [J].Electrical Automation , 2013,42 ( 05 ) : 183-185. 